\title{
The Role of Suppression in the Upward Spread of Masking
}

\author{
${\text { Ifat } \text { Yasin }^{1} \text { and Christopher J. Plack }}^{2}$ \\ ${ }^{1}$ Department of Experimental Psychology, University of Oxford, South Parks Road, Oxford, OX1 3UD, UK \\ ${ }^{2}$ Department of Psychology, University of Essex, Wivenhoe Park, Colchester, CO4 3SQ, UK
}

Received: 10 January 2005; Accepted: 11 August 2005; Online publication: 28 October 2005

\begin{abstract}
The upward spread of masking refers to the higher growth rate of masking for maskers lower in frequency than the signal, compared to maskers at the signal frequency (Wegel RL, Lane CE. The auditory masking of one pure tone by another and its possible relation to the dynamics of the inner ear. Physics Rev. 23:266-285, 1924; Egan JP, Hake HW. On the masking pattern of a simple auditory stimulus. J. Acoust. Soc. Am. 22:622-630, 1950; Delgutte B. Physiological mechanisms of psychophysical masking: Observations from auditory-nerve fibres. J. Acoust. Soc. Am. 87:791-809, 1990a, Delgutte B. Two-tone rate suppression in auditory-nerve fibres: Dependence on suppressor frequency and level. Hear Res. 49:225-246, 1990b). The upward spread of simultaneous masking may arise from a combination of excitatory and suppressive effects. In this study, growth of masking functions were obtained for a 4$\mathrm{kHz}$ signal masked by an on-frequency $(4 \mathrm{kHz})$ or offfrequency $(2.4 \mathrm{kHz})$, simultaneous or forward mask$\mathrm{er}$, in the presence of a notched noise with a center frequency of $4 \mathrm{kHz}$ presented to restrict off-frequency listening. Compression was estimated from the slopes of the off-frequency growth of masking functions. Suppression was estimated by comparing the offfrequency simultaneous- and forward-masked growth of masking functions. Results showed that, for midlevel signals (35-60 dB SPL), the compression exponent estimated from simultaneous and forward masking averaged 0.31 and 0.26 , respectively. The maximum amount of suppression (defined as the
\end{abstract}

Correspondence to: Ifat $\operatorname{Yasin}^{1} \cdot$ Department of Experimental Psychology - University of Oxford, South Parks Road, Oxford, OX1 3UD, UK. Telephone: (01865) 271334; fax: (01865) 310447; email: ifat.yasin@psy.ox.ac.uk decrease in the basilar-membrane response to the signal) was variable, ranging from about 6 to $17 \mathrm{~dB}$ across subjects. Despite the substantial reduction in the response to the signal, the results suggest that suppression has a minimal effect on the slope of the masking function at mid levels. Rather, upward spread of masking seems to be mainly determined by the compressive basilar-membrane response to the signal in relation to the linear response to the lowerfrequency masker.

Keywords: psychoacoustics, compression, suppression, masking

\section{INTRODUCTION}

For a signal presented with a lower-frequency masker, a given increase in masker level produces a greater increase in signal level at threshold (Wegel and Lane 1924; Egan and Hake 1950). This nonlinear growth of masking $(\mathrm{GOM})$ is commonly referred to as the upward spread of masking (USM). For simultaneous maskers and signals, USM may result from an upward spread of suppression (Wightman et al. 1977; Weber 1983; Fahey and Allen 1985; Costalupes et al. 1987; Delgutte 1990a), in which the reduction in the response to the signal increases with increasing masker level, and/or an upward spread of excitatory masking (Delgutte 1990a; Zwicker and Fastl 1990; Beveridge and Carlyon 1996; Moore and Vickers 1997; Oxenham and Plack 1998; Gorga et al. 2002), in which the response to the signal grows more slowly (compressively) with level than the response to the masker. Both phenomena are consequences of the frequency-dependent compressive nonlinearity on the basilar membrane (BM), produced by an active 
gain mechanism (Gold 1953; Kemp 1978) thought to be mediated by the outer hair cells (Brownwell et al. 1985).

The characteristics of cochlear nonlinearity can be measured in the response of the BM (Rhode 1971, 1977; Sellick et al. 1982; Robles et al. 1986; Ruggero et al. 1992; Rhode and Recio 2001). The active mechanism is responsible for the compressive growth of the $\mathrm{BM}$ response for a mid-level signal with a frequency close to the characteristic frequency $(\mathrm{CF})$ of the BM place of recording (Ruggero et al. 1997). In contrast, the BM response for a signal frequency of about $0.7 \mathrm{CF}$ or less grows linearly (slope close to 1 $\mathrm{dB} / \mathrm{dB}$; Ruggero et al. 1997). Compressive and suppressive responses have also been measured in auditory neurons (Cooper and Yates 1994; Delgutte 1990a; Pang and Guinan 1997). A compressive growth of response can be inferred from a comparison of neural rate-level functions for a signal at CF, and a signal well below CF (e.g., Cooper and Yates 1994). Delgutte (1990b) measured the level of a CFtone required to maintain a criterion discharge rate for an auditory nerve fiber, as a function of the suppressor level (a suppression growth function). The amount of suppression was found to average about $40-50 \mathrm{~dB}$; the $\mathrm{CF}$ tone had to be increased by about $40-50 \mathrm{~dB}$ (increase in level from 30 to $80 \mathrm{~dB}$ SPL) to maintain the discharge rate at criterion level, for an increase in suppressor level from about 60 to $90 \mathrm{~dB}$ SPL). Similarly high amounts of suppression were reported by Javel (1981); for a suppressor lower in frequency than the $\mathrm{CF}$, the shift of neural response functions (based on a measure of the synchrony of neural discharge) to higher levels was about $28 \mathrm{~dB}$.

Although nonlinear growth of suppression may be the major cause of USM for low-level signals (Delgutte 1990a), physiological (Robles et al. 1987; Ruggero et al. 1992) and psychophysical (Yasin and Plack 2003) studies suggest that for mid-level signals suppression may reduce the USM by increasing the slope of the BM I/O function and hence decreasing the compression in the signal response. Because suppression requires simultaneous presentation of the suppressor and suppressee (Arthur et al. 1971; Houtgast 1972, 1974; Fastl and Bechly 1983), simultaneous-masking experiments may underestimate the amount of compression that occurs in the absence of a suppressor (Stelmachowicz et al. 1987). In forward masking, however, the masker cannot suppress the signal and a more accurate estimate of compression can be obtained (Oxenham and Plack 1997; Nelson et al. 2001). Oxenham and Plack (1998) estimated compression by comparing the slopes of on- and offfrequency GOM functions and reported that compression estimates were slightly, but significantly, different for their simultaneous and nonsimulta- neous conditions (compression exponents of about 0.5 and 0.4 , respectively). The amount of suppression in simultaneous masking can be estimated by comparing the thresholds for a simultaneous- and forward-masked signal (Moore and Glasberg 1982; Moore and Vickers 1997; Oxenham and Plack 1998; Sommers and Gehr 1998). For example, Oxenham and Plack (1998) estimated the amount of suppression by taking the difference between the offfrequency simultaneous and nonsimultaneous masked signal thresholds.

There is a possibility that the compression and suppression estimated by some psychophysical studies may not be a true reflection of the actual amounts of compression and suppression as a result of offfrequency listening (Leshowitz and Wightman 1971; Johnson-Davies and Patterson 1979; O'Loughlin and Moore 1981). Off-frequency listening refers to the detection of signal activity from a region of the BM with a $\mathrm{CF}$ other than that of the signal. For example, if the masker frequency is below the signal frequency, then the signal may be detected using a place with a $\mathrm{CF}$ above the signal frequency. Because the response to a below-CF signal grows more linearly than the response to a signal at $\mathrm{CF}$, measures of compression without background noise may be underestimates (Nelson et al. 2001). As off-frequency listening decreases the estimate of compression, it is possible that it also affects the estimate of suppression. Oxenham and Plack (1998) found compression exponents of 0.4 and 0.5 estimated from off-frequency simultaneous- and forward-masked GOM slopes; however, they did acknowledge that the lack of a background noise may have affected the compression estimates. Indeed, these values are much higher (i.e., suggest less compression) than the estimate of 0.16 from forward-masked GOM functions derived with high-pass noise (Oxenham and Plack 1997). The use of a noise to restrict off-frequency listening in measurements of GOM has been evaluated by Nelson et al. (2001). Estimated compression was found to be greater with the notched noise than without; the notched noise restricted the use of signal information from areas adjacent to the signal place, where the signal response grows linearly. In summary, a comparison of GOM slopes measured in the absence of notched noise centered at the signal frequency may lead to an underestimate of the roles of both signal compression and suppression in USM.

The aim of the present study was to extend the findings of Oxenham and Plack (1998) by measuring the contributions of compression and suppression to USM in the presence of background notched noise designed to restrict off-frequency listening. GOM functions were obtained for a $4-\mathrm{kHz}$ signal masked by an on-frequency $(4 \mathrm{kHz})$ or off-frequency $(2.4$ 
$\mathrm{kHz}$ ) simultaneous or forward masker. The amount of signal compression was estimated from the slopes of the off-frequency GOM functions. Suppression was estimated by comparing the off-frequency simultaneous- and forward-masked GOM functions. Because of the need to keep the signal level constant relative to the level of the notched noise, the present study adaptively varied the masker level, rather than the signal level.

\section{METHODS}

\section{Stimuli}

Thresholds for a $4-\mathrm{kHz}$ pure-tone signal were obtained in the presence of a $500-\mathrm{Hz}$ wide noise masker (3-dB downpoints and attenuation slopes of $90 \mathrm{~dB} /$ octave) centered at either $4 \mathrm{kHz}$ (on-frequency) or $2.4 \mathrm{kHz}$ (off-frequency). The signal had no steady-state duration and 3-ms raised-cosine on- and off-set ramps. The masker had a steady-state duration of $200 \mathrm{~ms}$ and 2-ms raised-cosine on- and off-set ramps. The signal was presented in the temporal center of the masker (simultaneous masking) or at a masker-signal gap (ramp offset to ramp onset) of 6 ms (forward masking). The signal was presented at levels between 20 and $80 \mathrm{~dB}$ SPL in 5-dB steps. A notched noise was added to limit off-frequency masking by Oxenham and Plack (1997). In their study, a notched noise was presented with a spectrum level $30 \mathrm{~dB}$ below the spectrum level needed to mask the signal for one of the subjects. The spectrum level in the passband was about 50-60 $\mathrm{dB}$ less than the signal level in dB SPL. In the present study, the notched noise was presented with a spectrum level in the passband $40 \mathrm{~dB}$ less than the signal level, although the signal was still clearly audible. The notched noise had a center frequency of $4 \mathrm{kHz}$, a notch width of $1 \mathrm{kHz}$ (3- $\mathrm{dB}$ downpoints), and attenuation slopes of $90 \mathrm{~dB} /$ octave. The noise was gated on $50 \mathrm{~ms}$ before masker onset and gated off 50 ms after signal offset. All stimuli were digitally generated by a PC using a sampling rate of $48 \mathrm{kHz}$ and output via a soundcard with 24-bit resolution. Antialiasing was provided by built-in filters. The stimuli were presented to the right channel of Sennheiser HD 580 headphones. The headphone input came directly from the output of the soundcard DAC.

\section{Procedure}

Subjects were tested individually while seated in an IAC double-walled sound-attenuating booth. The stimuli were presented to the subjects' right ears. A 3I-3AFC adaptive procedure was used to determine the masker level at which the subject would achieve $70.7 \%$ correct on the psychometric function (Levitt 1971). A block of trials began with the presentation of a light on a computer-simulated response box. Subjects started a block of trials by pressing a start key. The length of each observation interval was indicated by a light on the response box in one of two rectangles. The interstimulus interval was $500 \mathrm{~ms}$. On each trial, the masker was presented in all three intervals. The signal was presented at random in one of the intervals. The task within each trial was to select the signal interval. Subjects responded by pressing the appropriate response key. After a response, visual feedback was provided by the presentation of a colored light. In each block of such trials, the masker level was decreased after an incorrect response and increased after every two consecutive correct responses. A reversal was counted every time the masker level changed direction. The masker level was varied in steps of $4 \mathrm{~dB}$ for the first four reversals. For the following 12 reversals the step size was reduced to $2 \mathrm{~dB}$ and the levels for the last 12 reversals were averaged to obtain the threshold value. In this way, an estimate of threshold was obtained from each block of trials. Data were collected after 2 $\mathrm{h}$ of practice. Five estimates of threshold were obtained for each condition. The most deviant threshold was discarded and the mean was calculated from the remaining four estimates of threshold. The maximum masker spectrum level that could be produced by the system without clipping was $72 \mathrm{~dB}$. If a masker spectrum level of $72 \mathrm{~dB}$ was reached within a block of trials, the estimate of threshold from that block was discarded. Temporal (simultaneous and nonsimultaneous) and frequency (on- and off-frequency) conditions of the masker were presented as a randomized set.

\section{Subjects}

Four subjects were tested. One was the author (I.Y.) and the other three subjects (A.B., C.O., and P.P.) were paid for their services. Absolute thresholds for the $4-\mathrm{kHz} 6-\mathrm{ms}$ signal used in the experiment were 10.4, 13.4, 19.4, and 9.8 dB SPL, respectively.

\section{RESULTS}

\section{GOM functions for simultaneous or forward maskers}

The individual GOM functions for both forward- and simultaneous-masking conditions are shown in Figure 1. Each panel presents a set of GOM functions for an individual subject. Filled and open squares represent the on-frequency simultaneous- and for- 


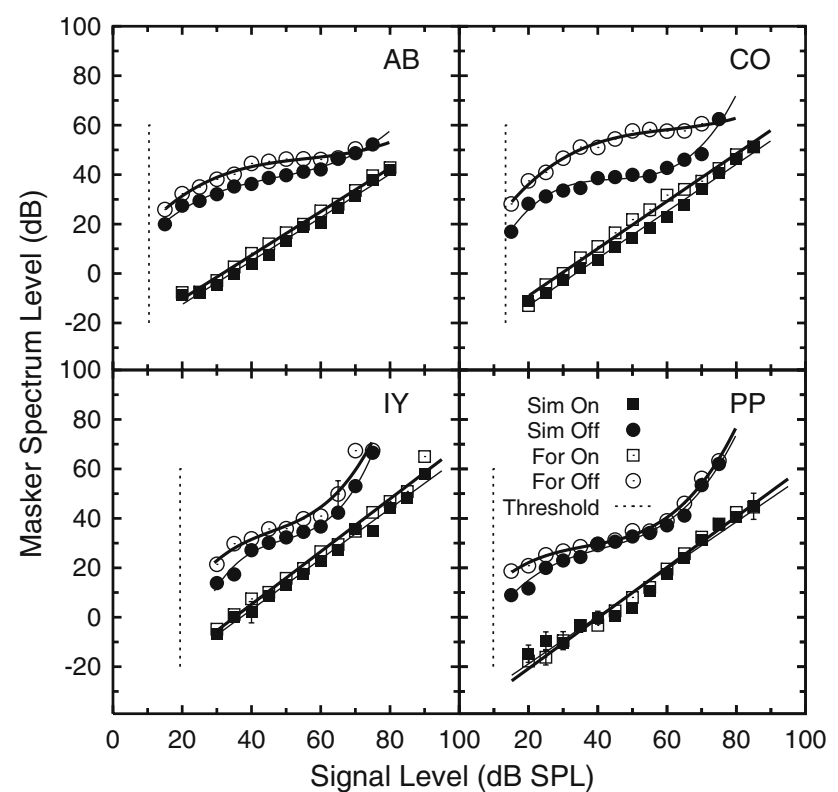

FIG. 1. GOM functions for individual subjects. Each panel shows masker levels as a function of signal level for on- and off-frequency simultaneous and forward maskers. On-frequency simultaneousand forward-masked GOM data are represented by filled and open squares respectively. Off-frequency simultaneous- and forwardmasked GOM data are represented by filled and open circles, respectively. Dashed lines represent absolute threshold for the signal alone. Solid thick and thin lines represent functions fitted to the forward- and simultaneous-masked data, respectively. Error bars smaller than the data point symbols are omitted for clarity.

ward-masked GOM data, respectively. Filled and open circles represent the off-frequency simultaneous- and forward-masked GOM data, respectively. Dashed lines represent threshold values for the signal alone. Within each panel, the on-frequency simultaneous and forward-masked GOM data were fit with linear functions and the off-frequency simultaneousand forward-masked GOM data were fit by thirdorder polynomial functions. In both cases, the fitting procedure minimized the root mean square $(\mathrm{rms})$ deviations between the experimental and predicted values. The solid thick and thin lines fitted to the data represent the functions fitted to the forwardand simultaneous-masked data, respectively. Consistent with the results of Oxenham and Plack (1997, 1998), for all subjects the increase in the onfrequency masker level with increasing signal level is close to linear (mean gradient, $0.96 \mathrm{~dB} / \mathrm{dB}$ ). On average, there was a slight tendency for the onfrequency forward-masked functions to be higher than the simultaneous functions (mean of $2.4 \mathrm{~dB}$ across listeners), with subject C.O. showing the greatest difference $(4.1 \mathrm{~dB})$. The off-frequency simultaneous- and forward-masked functions diverge for all subjects, with generally higher masker levels required for threshold with an off-frequency forward masker than an off-frequency simultaneous masker. The range of signal levels over which this offfrequency divergence occurs and the extent of the divergence differ across subjects. For A.B., C.O. and I.Y., the divergence occurs for a relatively large range of signal levels between about 30 and $70 \mathrm{~dB}$ SPL, with a greater overall divergence for C.O. For P.P., the divergence occurs for a smaller and lower range of signal levels, between about 20 and $30 \mathrm{~dB}$ SPL. For most subjects, the slope of the off-frequency, simultaneous- and forward-masked GOM function returns to a value of $1 \mathrm{~dB} / \mathrm{dB}$ (or greater) for higher signal levels (above about $60 \mathrm{~dB}$ SPL).

\section{Estimates of compression}

Following Oxenham and Plack (1997), the GOM function for the off-frequency masker may be assumed to be an estimate of the shape of the BM response function for the signal at the place tuned to the signal. The slope of the off-frequency GOM function is an estimate of the compression exponent, $c$. The value of $c$ was calculated by taking the first derivative of the third-order polynomial fits to the offfrequency simultaneous- and forward-masked GOM data. The derived values of $c$, plotted as a function of signal level, are shown in Figure 2. Each panel of Figure 2 presents two compression functions for a given subject, with thin and thick lines representing values of $c$ for a simultaneous or forward masker, respectively. Compression estimates $(1 / c)$ are low for low-level signals for all subjects with either a simultaneous or forward masker. Compression estimates are also low for high-level signals with a simultaneous

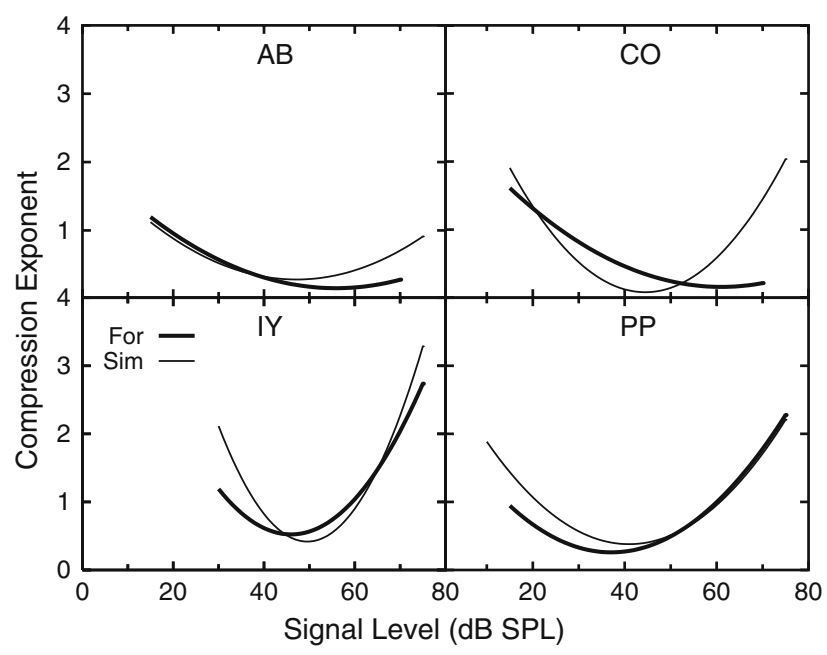

FIG. 2. Compression functions for individual subjects. Each panel shows the value of $c$ as a function of signal level. Thin and thick lines represent values of $c$ for a simultaneous (Sim) and forward masker (For), respectively. 


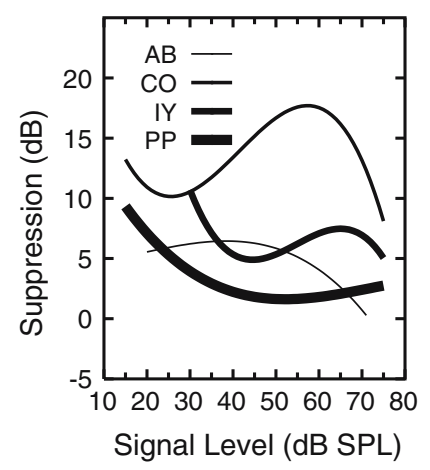

FIG. 3. The amount of suppression as a function of signal level. Lines of differing thickness represent individual subject data.

masker for all subjects and a forward masker for I.Y. and P.P. The estimates of $c$ greater than 1 , as seen in the high-level off-frequency data for C.O., I.Y., and P.P. are puzzling. Variability may be partly responsible: The polynomial fits were pushed into the final steep phase largely by the highest one or two data points.

For all subjects, compression is greatest for midlevel signals (35-60 dB SPL), for either a simultaneous or forward masker. For mid-level signals, maximum forward-masked compression exponents average 0.26 across the four subjects, with compression exponents for subjects A.B. and C.O. of about 0.17 . Over the same range, the simultaneous-masked compression exponents average 0.31 . However, there are differences between individuals. The estimates of signal compression are greater for a forward than a simultaneous masker for A.B. and P.P., similar for both maskers for I.Y., and greater for a simultaneous than a forward masker for C.O. For relatively highlevel signals of about 55-70 dB SPL for A.B. and C.O., the compression estimates are greater for forward than for simultaneous masking. For low-level signals (15-40 dB SPL for P.P. and 30-45 dB SPL for I.Y.), the compression estimates are greater for forward than simultaneous masking. For I.Y. for mid-level signals (45-60 dB SPL), there is slightly more compression in simultaneous than forward masking. However, in the case of the off-frequency forwardmasking thresholds for this subject, the slope of the best-fitting polynomial seems to be slightly greater than the slope inherent in the data at mid levels.

\section{Estimates of suppression}

To estimate the amount of suppression as a function of signal level, the third-order polynomial functions fitted to the off-frequency data were used to generate values of masker levels for a range of signal levels. The amount of suppression for a given signal level was calculated by subtracting the interpolated masker level for the off-frequency simultaneous condition from the masker level for the off-frequency forward condition. The values of suppression calculated in this manner indicate the decrease in the BM response to the signal at the signal place. Because the off-frequency masker threshold can be taken as an estimate of the $\mathrm{BM}$ response to the signal (Oxenham and Plack 1997), the decrease in the physical level of the off-frequency masker from a forward- to simultaneous-masking condition is assumed to be equivalent to the decrease in the BM response to the suppressed signal.

The amount of suppression as a function of signal level is shown in Figure 3. The maximum amount of suppression ranges from about 6 to $17 \mathrm{~dB}$ across subjects. For A.B. and C.O., suppression increases from low signal levels to a maximum of about 6 and $17 \mathrm{~dB}$, respectively, then decreases for higher signal levels. For I.Y. and P.P., the amount of suppression has a maximum of about $10 \mathrm{~dB}$ at relatively low signal levels.

\section{DISCUSSION}

The growth of masking and compression

Despite individual differences, there are two main consistent features of the data. First, the on-frequency simultaneous- and forward-masked GOM functions are linear. Previous studies with a brief signal and a short masker-signal gap have also reported linear onfrequency forward-masked GOM functions (Oxenham and Moore 1995; Oxenham and Plack 1997, 1998; Plack and Oxenham 1998). Linear masking functions can be explained by the equivalent rate of $\mathrm{BM}$ response growth for both the on-frequency masker and the signal at the signal place (Plack and Oxenham 1998); the BM response to both the masker and signal grows either linearly (for low-level signals) or compressively (for mid-level signals). The linear increase in level of the simultaneous onfrequency masker with increase in signal level was similar to that reported by Oxenham and Plack (1998; 10-ms 4-kHz signal, 500-Hz noise masker). However, Oxenham et al. (1997) reported that for short (2 ms), 5-kHz signals, signal thresholds do not increase linearly for mid-level increases in a simultaneously presented broadband noise masker; a greater signal level increase is required for mid-level masker levels than for very low or high masker levels. Similarly, in van Klitzing and Kohlrausch's (1994) study, for a 2-ms signal masked by a simultaneous frozen-noise masker, a nonlinear response was particularly evident for mid-level maskers; a greater increase in masked threshold was observed for an increase in masker level from 30 to $50 \mathrm{~dB}$ than for an 
increase in masker level from 50 to $70 \mathrm{~dB}$. A linear increase in level of the simultaneous on-frequency masker with increase in signal level in the present study is most likely explained by the use of a narrowband noise masker, the internal representation of which grows at a similar rate to that of the signal, with the effect that for mid-level signals, compression acts to reduce the representations of the masker and signal equally. It appears unlikely that signal splatter facilitated signal detection in the present study, due to the use of the notched noise.

Second, both off-frequency forward- and simultaneous-masked GOM functions are nonlinear for midlevel signals, consistent with previous studies (Oxenham and Plack 1997, 1998). The nonlinear GOM for mid-level signals is explained by a compressive signal response growth with respect to a linear masker response growth at the signal place (Rhode 1971; Sellick et al. 1982; Robles et al. 1986; Yates et al. 1990; Nelson and Schroder 1997; Oxenham and Plack 1997; 1998; Nelson et al. 2001). For all subjects, the maximum compression for either a simultaneous or forward masker occurred for signals around 35-60 dB SPL. According to physiological data, it is for this range of signals that the growth of excitation is nonlinear at the signal place (Yates et al. 1990; Murugasu and Russell 1995). Maximum forwardmasked compression exponents averaged 0.26 with compression exponents for subjects A.B. and C.O. close to 0.17 . An estimate of 0.17 is comparable to the compression exponent of 0.16 derived from GOM functions with high-pass noise (Oxenham and Plack 1997; Nelson et al. 2001). These higher compression estimates with notched or high-pass noise are consistent with the higher compression estimates from animal data (Sellick et al. 1982; Yates et al. 1990; Ruggero et al. 1992). The average compression exponent estimate of 0.26 is comparable to the compression exponent of 0.3 from $\mathrm{BM} \mathrm{I} / \mathrm{O}$ functions derived from forward-masking thresholds with notched noise (Yasin and Plack 2003). Oxenham and Plack (1998) reported compression exponents derived from nonsimultaneous GOM functions to average 0.43 across subjects, for the same signal and masker frequencies as used in this study but without the notched noise to restrict off-frequency listening. The present results suggest that the lower estimate of compression in their study may have been due to off-frequency listening. The BM response basal to the signal place grows more linearly than the response at the signal place, hence the use of information from a more basal location may linearize the GOM, so increasing the compression exponent estimate.

For most subjects the slope of the off-frequency GOM function becomes much steeper at higher sig- nal levels (above about 60 dB SPL). Some (Ruggero and Rich 1991; Ruggero et al. 1992; Oxenham and Plack 1997; Gregan et al. 1998; Bacon et al. 1999; Nelson et al. 2001; Yasin and Plack 2003), although not all (Bacon and Viemeister 1985; Murugasu and Russell 1995; Ruggero et al. 1997) physiological and psychophysical studies show that the slope of the I/O function or GOM function returns to a value of 1 $\mathrm{dB} / \mathrm{dB}$ for high-level signals. The implication being that for high-level signals the BM response to both the signal and masker may grow linearly at the signal place, as is the case for low-level signals (Delgutte 1990a; Yates et al. 1990). A return to linearity at high levels could also be explained by the level-dependent shift of the peak of the signal excitation pattern toward the basal region of the BM (McFadden and Yama 1983). At high levels, the peak of the traveling wave grows more linearly than the CF response (e.g., Ruggero et al. 1997). Another possibility is that intense masker levels activate the middle ear reflex. The middle-ear reflex refers to the contraction of the stapedius muscle in response to intense sounds, resulting in the attenuation of the sound level reaching the cochlea (Nuttal 1974). As contraction of the stapedius muscle attenuates low frequencies more than high frequencies (Kobler et al. 1992), the effect of the reflex may have been to increase the off-frequency masker level required at high levels. However, because the reflex has little effect on frequencies above 2 kHz (e.g., Rosowski and Relkin 2001), this seems unlikely to be a complete explanation.

For some subjects, the compression estimates for higher signal levels suggest an expansive nonlinearity, i.e., a growth of response at the signal place of greater than $1 \mathrm{~dB} / \mathrm{dB}$. However, only one physiological study (Zinn et al. 2000) clearly suggests an expansive nonlinearity, at the apical end of the guinea-pig cochlea (a CF of around 300-400 Hz). It is possible that the high-level expansion observed in the present data was attributable to a combination of the basalward shift and the middle-ear reflex. In some cases, expansion was also observed at low levels. The dashed line in Figure 1 represents absolute threshold for the 6-ms signal presented in the experiment. The expansive behavior of the GOM functions close to absolute threshold may be due to the influence of the internal noise floor that limits detectability for signals in quiet. This reduces the level of the masker needed for the lowest signal levels.

In the present study, the on-frequency forwardmasked thresholds were on average $2.4 \mathrm{~dB}$ greater than the on-frequency simultaneous-masked thresholds. That is, the signal required on average $2.4 \mathrm{~dB}$ more masking when presented with an on-frequency forward masker than when presented with an on- 
frequency simultaneous masker. Using similar parameters, Oxenham and Plack (1998) also found a tendency for the nonsimultaneous-masked thresholds to be generally greater than the simultaneousmasked thresholds (as represented in terms of the GOM axes of the present study), with an overall difference across subjects of $2.1 \mathrm{~dB}$, which is similar to the mean difference found in this study. Some previous studies with short masker-signal intervals have shown the difference between simultaneousand forward-masked signal thresholds to be greater than the mean difference of $2.4 \mathrm{~dB}$ difference in masker thresholds demonstrated in the present study. These studies showed forward-masked signal thresholds to be greater than simultaneous-masked thresholds by about $20 \mathrm{~dB}$ (as represented in terms of the GOM axes of the present study) (Gralla 1992: 1.5-ms 4-kHz signal, broadband masker, masker-signal delay of $8.5 \mathrm{~ms}$; Dau et al. 1996: 10-ms, $1-\mathrm{kHz}$, signal, frozen-noise masker, masker-signal delay of $10 \mathrm{~ms}$ ). However, the magnitude of the difference between forward- and simultaneous-masked thresholds depends on a number of factors such as the temporal relationship between signal and masker, masker level, and type of masker. For instance, in the studies conducted by both Gralla (1992) and Dau et al. (1996), the masker bandwidth was much greater than the bandwidth of the auditory filter (Glasberg and Moore, 1990) centered at the signal frequency, whereas in the present study the masker bandwidth was similar to the bandwidth of the filter centered at the signal frequency (noise-masker bandwidth: 500 $\mathrm{Hz}$, filter bandwidth $456 \mathrm{~Hz}$ ). Lee and Bacon (1988) showed that for a masker-signal gap of $0 \mathrm{~ms}$, the mean masked thresholds for a $20-\mathrm{ms} 4-\mathrm{kHz}$ signal in the presence of a broadband masker were about 20 $\mathrm{dB}$ lower than the masked thresholds in the presence of an ERB-width masker. When the masker-signal gap between masker and signal was increased to $20 \mathrm{~ms}$, the mean masked thresholds for the signal in the presence of a broadband masker was about $10 \mathrm{~dB}$ lower than the masked thresholds with an ERB-width masker. The broadband noise acts to suppress the internal representation of the masker falling within the filter bandwidth thereby decreasing signal thresholds. In line with this, Dubno and Ahlstrom (2001) showed that the difference between simultaneousand forward-masked thresholds is greater for a noise masker with a wide bandwidth than a noise with a narrow bandwidth. For a signal of $2 \mathrm{kHz}$ (filter bandwidth approximately $240 \mathrm{~Hz}$ ) and a masker with a bandwidth of $400 \mathrm{~Hz}$, the mean difference between simultaneous and forward masked thresholds was small, around $5 \mathrm{~dB}$, but this difference increased to about $35 \mathrm{~dB}$ for a masker bandwidth of $2 \mathrm{kHz}$. In addition, for signal thresholds measured as a func- tion of masker bandwidth, the forward-masked thresholds decreased faster then the simultaneousmasked thresholds as the bandwidth of the masker was increased (Dubno and Ahlstrom 2001), with the simultaneous-masked thresholds always greater than the forward-masked thresholds. As Dubno and Ahlstrom used a signal close to the onset of the masker, the simultaneous-masked thresholds would be anticipated to be slightly greater due to overshoot (van Klitzing and Kohlrausch 1994). If this is the case, then it might be supposed that the difference between simultaneous and forward masked signal thresholds might be even smaller for a signal simultaneously presented in the temporal center of the masker. In sum, the relatively small difference between the on-frequency simultaneous and forwardmasked thresholds in this study may be due to three main factors: a short masker-signal interval, a narrowband noise masker bandwidth close to that of the filter centered on $4 \mathrm{kHz}$, and temporal positioning of the signal at the center, rather than near the onset of the masker.

\section{The effects of suppression}

The maximum amount of suppression estimated in this study ranged from about 6 to $17 \mathrm{~dB}$ across subjects, with an average value for maximum suppression of about $10 \mathrm{~dB}$. The results show great intersubject variation in the estimated suppression, as has previously been noted for both human (e.g., Oxenham and Plack 1998) and animal (e.g., Ruggero et al. 1992) data. The fact that this variability is also found in the physiology suggests that it may be a consequence of differences in the cochlear response itself, rather than a consequence of measurement variability. Oxenham and Plack (1998) estimated suppression from GOM functions (using the same signal and masker frequencies as in this study) by subtracting the difference between the on-frequency simultaneous and nonsimultaneous GOM functions from the difference between the two off-frequency functions at each signal level. Maximum suppression seemed to occur for signal levels around 40-60 dB SPL (close to the mid-level range of signal levels of 35-60 dB SPL observed in this study) and maximum suppression ranged from 15 to $32 \mathrm{~dB}$, with an average estimate of around $20 \mathrm{~dB}$. It is important to note, however, that estimating suppression as the change in signal threshold produces a larger value than estimating suppression as the change in off-frequency masker threshold (as used here). While the former measures the change in the physical signal level needed to overcome the suppression, the latter estimates the change in the BM response produced by the signal. Because the signal is compressed, the 
former will always be greater than the latter. Measuring suppression as a change in signal level (i.e., the horizontal difference between the forward and simultaneous off-frequency GOM functions in Figure 1) yields an average value for maximum suppression of about $18 \mathrm{~dB}$ for the present study. In other words, the suppression measured here is similar to that reported by Oxenham and Plack, although less than that reported by the physiological studies described in the Introduction. Other psychophysical studies measuring suppression as a change in signal threshold have also found variable amounts of suppression up to about 30-35 dB (Duifhuis 1980; Shannon 1986). Both Duifhuis (1980) and Shannon (1986) estimated suppression using pulsation threshold and found suppression to range from 12 to $35 \mathrm{~dB}(600 \mathrm{~Hz}$ suppressor, $1 \mathrm{kHz}$ suppressee) and from 20 to $30 \mathrm{~dB}$ (400 Hz suppressor, $1 \mathrm{kHz}$ suppressee), respectively. It appears that the amount of suppression is highly dependent on both the frequency and level relationship between the suppressor and suppressee (Duifhuis 1980).

If it is assumed that the growth of suppression contributes to USM at the signal place for low-level signals (Delgutte 1990a; Gorga et al. 2002), then the simultaneous GOM slopes may be expected to be shallower than those for forward masking, as is the case for C.O. for low-level signals. In this case, a shallower slope would not indicate compression but suppression of the signal. However, in the case of I.Y. and P.P. the reverse appears to be the case; shallower slopes are observed in forward rather than in simultaneous masking. The results of P.P. and I.Y. for low-level signals are compromised by the lack of a sufficient number of data points for signal levels below $40 \mathrm{~dB}$ SPL in order to derive meaningful estimates of compression. Also, when the signal level is close to the absolute threshold, the masking may rely more on internal noise rather than masker excitation. For the range of signals $40-60 \mathrm{~dB}$ SPL for which the BM response is known to be most compressive (e.g., Yates et al. 1990; Murugasu and Russell 1995), the estimates of signal compression are greater for a forward than a simultaneous masker for A.B. and P.P., similar for both maskers for I.Y., and greater for a simultaneous than a forward masker for C.O. However, in the case of I.Y., the slope of the polynomial seems to be a slight overestimate of the slope in the raw data. For mid-level signals suppression may steepen the compressive $\mathrm{BM}$ response to the signal (Ruggero et al. 1992; Yasin and Plack 2003), possibly by driving the compressive nonlinearity at the outer hair cell into saturation (Geisler and Sinex 1980; Javel 1981; Geisler 1985; Deng and Geisler 1985; Robles et al. 1986; Cheatham and Dallos 1989; Nuttal and Dolan 1993; Rhode and
Recio 2001; Geisler et al. 1990). Suppression may operate to reduce the nonlinear gain of the cochlear amplifier at the signal place in a level-dependent manner, thereby decreasing the compression (Robles et al. 1987; Ruggero et al. 1992; Rhode and Recio 2001). This effect is seen in the data for A.B. and C.O.

Pang and Guinan (1997) suggested that if the BM response to a signal grows with signal level with a slope of $c$, then the response generated by $1-\mathrm{dB}$ increase in a lower-frequency masker will reduce the gain at the signal place (suppress the signal) by $(1 / c)$ $-1 \mathrm{~dB}$. Plotted as signal level against masker level, the slope $(1 / c)$ of the GOM function in the suppressed condition should be one less than the slope of the GOM function in the unsuppressed condition. A decrease in slope by only 0.3 in the suppressed condition is suggested by the findings of Oxenham and Plack (1998); the slope of the off-frequency simultaneous GOM function was $2.0(c=0.5)$, whereas the slope of the off-frequency forwardmasked GOM function was $2.3(c=0.43)$. In the present study, plotted as signal level against masker level, the average slope of the off-frequency forwardmasked GOM function was $3.9(c=0.26)$, whereas the average slope of the off-frequency forward-masked GOM was $3.2(c=0.31)$. This gives an average reduction of the slope by about 0.6 in the presence of suppression. This value is closer to the reduction of the GOM slope by one suggested by Pang and Guinan.

Overall, it appears that for mid-level signals suppression only has a small effect on the USM. The similarity in the compression estimates for simultaneous and forward masking in the present study suggest that, although suppression reduces the response to the signal, suppression by a masker that varies with the signal level does not have a large effect on the slope of the response function. This implies that the gain of the cochlear amplifier is reduced, but that the slope of the function relating gain to input level is not greatly affected.

\section{CONCLUSIONS}

Estimates of compression in the upward spread of masking derived with notched noise to limit offfrequency listening are consistent with the high estimates of compression found in physiological (Sellick et al. 1982; Yates et al. 1990; Ruggero et al. 1992) and psychophysical (Nelson et al. 2001) studies. This is true both for nonsimultaneous and for simultaneous masking. The similarity between the slopes of the growth of masking functions for nonsimultaneous and simultaneous maskers suggests 
that the role of suppression in the upward spread of masking is relatively small for mid-level signals, consistent with the results of Oxenham and Plack (1998) and the theoretical analysis of Pang and Guinan (1997).

\section{ACKNOWLEDGMENTS}

This research was supported by EPSRC Grant GR/N07219. The first author was supported by a studentship from the ESRC. The authors thank Andrew Oxenham, an anonymous reviewer, and the editor for constructive comments on an earlier version of the manuscript.

\section{REFERENCES}

Arthur RM, Pfeiffer RR, Suga N. Properties of two-tone inhibition in primary auditory neurons. J. Physiol. 212:593-609, 1971.

BACON SP, VIEMEISTER NF. Temporal modulation transfer functions in normal-hearing and hearing-impaired listeners. Audiology 24:117-134, 1985 .

Bacon SP, Boden LN, Lee J, Repovsch JL. Growth of simultaneous masking for $f_{\mathrm{m}}<f_{\mathrm{s}}$ : effects of overall frequency and level. J. Acoust. Soc. Am. 106:341-350, 1999.

Beveridge HA, Carlyon RP. Effects of aspirin on human psychophysical tuning curves in forward and simultaneous masking. Hear. Res. 99:110-118, 1996.

Brownwell WE, Bader CR, Bertrand D, de Ribaupierre Y. Evoked mechanical responses of isolated cochlear outer hair cells. Science 227:194-196, 1985.

Cheatham MA, Dallos P. Two-tone suppression in inner hair cell responses. Hear. Res. 40:187-196, 1989.

COOPER NP, YATES GK. Nonlinear input-output functions derived from the responses of guinea-pig cochlear nerve fibre: variations with characteristic frequency. Hear Res. 78:221-234, 1994.

Costalupes J, Rich N, Ruggero M. Effects of excitatory and nonexcitatory suppressor tones on two-tone rate suppression in auditory nerve fibers. Hear. Res. 26:155-164, 1987.

Dau T, Púschel D, Kohlrausch A. A quantitative model of the "effective" signal processing in the auditory system. II. Simulations and measurements. J. Acoust. Soc. Am. 99:3623-3631, 1996.

Delgutte B. Physiological mechanisms of psychophysical masking: observations from auditory-nerve fibres. J. Acoust. Soc. Am. 87:791-809, 1990a.

Delgutte B. Two-tone rate suppression in auditory-nerve fibres: dependence on suppressor frequency and level. Hear Res. 49:225-246, 1990b.

Deng L, Geisler CD. Changes in the phase of excitatory-tone responses in cat auditory nerve fibers by suppressor tones and fatigue. J. Acoust. Soc. Am. 78:1633-1643, 1985.

Dubno JR, AhLstrom JB. Forward- and simultaneous-masked thresholds in band-limited maskers in subjects with normal hearing and cochlear hearing loss. J. Acoust. Soc. Am. 110:1049-1057, 2001.

Duifhuis H. Level effects in psychophysical two-tone suppression. J. Acoust. Soc. Am. 67:914-927, 1980.

EGAN JP, HAKE HW. On the masking pattern of a simple auditory stimulus. J. Acoust. Soc. Am. 22:622-630, 1950.

Fahey P, Allen JB. Nonlinear phenomena as observed in the ear canal and at the auditory nerve. J. Acoust. Soc. Am. 77:599-612, 1985.

Fastl H, Bechly M. Suppression in simultaneous masking. J. Acoust. Soc. Am. 74:754-757, 1983.

GEISLER C. Effects of a compressive nonlinearity in a cochlear model. J. Acoust. Soc. Am. 78:257-260, 1985.

GeIsLeR CD, Sinex DG. Responses of primary auditory fibres to combined noise and tonal stimuli. Hear. Res. 3:317-334, 1980.

Geisler C, Yates G, Patuzzi R, Johnstone B. Saturation of outer hair cell receptor currents causes two-tone suppression. Hear. Res. 44:241-256, 1990.

Glasberg BR, Moore BCJ. Derivation of auditory filter shapes from notched-noise data. Hear. Res. 47:103-138, 1990.

GoLD T. Hearing. IEEE Trans. Inf. Theory I:125-127, 1953.

Gorga MP, Neely ST, Dom PA, Dierking D. Evidence of upward spread of suppression in dpoae measurement. J. Acoust. Soc. Am. 112:2910-2920, 2002.

Gralla G. Wahrnehmungskriterien bei simultanund Nachhörschwellenmessungen. Acustica 77:243-251, 1992.

Gregan MJ, Bacon SP, LeE J. Masking by sinusoidally amplitudemodulated tonal maskers. J. Acoust. Soc. Am. 103:1012-1021, 1998.

Houtgast T. Psychophysical evidence for lateral inhibition in hearing. J. Acoust. Soc. Am. 51:1885-1894, 1972.

Houtgast T. Lateral suppression in hearing. PhD thesis. Free University of Amsterdam, 1974.

JAVEL E. Suppression of auditory nerve responses I: temporal analysis, intensity effects and suppression contours. J. Acoust. Soc. Am. 69:1735-1745, 1981.

Johnson-Davies D, Patterson RD. Psychophysical tuning curves: restricting the listening band to the signal region. J. Acoust. Soc. Am. 65:765-770, 1979.

KemP DT. Stimulated acoustic emissions from within the human auditory system J. Acoust. Soc. Am. 64:1386-1391, 1978.

Kobler JB, Guinan JJ Jr, Vacher SR, Norris BE. Acoustic reflex frequency selectivity in single stapedius motoneurons of the cat. J. Neurophysiol. 68:807-817, 1992.

LEe J, BACON SP. Psychophysical suppression as a function of signal frequency: noise and tonal maskers. J. Acoust. Soc. Am. 104:1013-1022, 1988.

Leshowitz B, Wightman FL. On-frequency masking with continuous sinusoids. J. Acoust. Soc. Am. 49:1180-1190, 1971.

LevitT H. Transformed up-down methods in psychoacoustics. J. Acoust. Soc. Am. 49:467-477, 1971.

McFadden D, Yama MF. Upward shifts in the masking pattern with increasing masker intensity. J. Acoust. Soc. Am. 74:1185-1189, 1983.

Moore BCJ, Glasberg B. Interpreting the role of suppression in psychophysical tuning curves. J. Acoust. Soc. Am. 72:1374-1379, 1982.

Moore BCJ, Vickers DA. The role of spread excitation and suppression in simultaneous masking. J. Acoust. Soc. Am. 102:2284-2290, 1997.

Murugasu E, Russell IJ. Salicylate ototoxicity: the effects on the basilar membrane displacement, cochlear microphonics, neural responses in the basal turn of the guinea pig cochlea. Aud. Neurosci. 1:139-150, 1995.

Nelson DA, Schroder AC. Linearized response growth inferred from growth-of-masking slopes in ears with cochlear hearing loss. J. Acoust. Soc. Am. 101:2186-2201, 1997.

Nelson D, Schroder A, Wojtczak M. A new procedure for measuring peripheral compression in normal-hearing and hearing-impaired listeners. J. Acoust. Soc. Am. 110:2045-2064, 2001. 
Nuttal AL. Tympanic muscle effects on middle-ear transfer characteristic. J. Acoust. Soc. Am. 56:1239-1247, 1974.

NutTal AL, Dolan DF. Two-tone suppression of inner hair cell and basilar membrane responses in the guinea pig. J. Acoust. Soc. Am. 93:390-400, 1993.

O’Loughlin B, Moore B. Off-frequency listening: effects on psychoacoustical tuning curves obtained in simultaneous and forward masking. J. Acoust. Soc. Am. 69:1119-1125, 1981.

Oxenham AJ, Moore BCJ. Additivity of masking in normally hearing and hearing-impaired subjects. J. Acoust. Soc. Am. 98:19211934, 1995 .

Oxenham AJ, Plack CJ. A behavioral measure of basilar-membrane nonlinearity in listeners with normal and impaired hearing. J. Acoust. Soc. Am. 101:3666-3675, 1997.

Oxenham AJ, Plack CJ. Suppression and the upward spread of masking. J. Acoust. Soc. Am. 104:3500-3510, 1998.

Oxenham AJ, Moore BCJ, Vickers DA. Short-term temporal integration: evidence for the influence of peripheral compression. J. Acoust. Soc. Am. 101:3676-3687, 1997.

PANG XD, GUinan JJ JR. Growth rate of simultaneous masking in cat auditory-nerve fibers: relationship to the growth of basilarmembrane motion and the origin of two-tone suppression. J. Acoust. Soc. Am. 102:3564-3575, 1997.

Plack CJ, Oxenham AJ. Basilar-membrane nonlinearity and the growth of forward masking. J. Acoust. Soc. Am. 103:1598-1608, 1998.

RHODE W. Observations of the vibration of the basilar membrane in squirrel monkeys using the Mossbauer technique. J. Acoust. Soc. Am. 49:1218, 1971.

RHoDE WS. Some observations on two-tone interactions measured with the Mossbauer effect. In: Evans EF and Wilson JP (eds) Psychophysics and Physiology of Hearing. Academic Press, London, pp 27-38, 1977.

Rhode W, Recio A. Multicomponent stimulus interactions observed in basilar-membrane vibration in the basal region of the chinchilla cochlea. J. Acoust. Soc. Am. 110:3140-3154, 2001.

Robles L, Ruggero MA, Rich N. Basilar membrane mechanics at the base of the chinchilla cochlea. I. Input-output functions, tuning curves, and response phases. J. Acoust. Soc. Am. 80:1364-1374, 1986.

Robles L, Ruggero MA, Rich NC. Two-tone suppression in the basilar membrane of the chinchilla. Abstracts of the Second World Congress of Neuroscience. Neuroscience 22:S727, 1987.

Rosowski JJ, RELKIN EM. Introduction to the analysis of middle ear function. In: Jahn AF and Santos-Sacchi J (eds) Physiology of the Ear. Singular, San Diego, pp 161-190, 2001.

Ruggero M, Rich N. Furosemide alters organ of Corti mechanics: evidence for feedback of outer hair cells upon the basilar membrane. J. Neurosci. 11:1057-1067, 1991.

Ruggero M, Robles L, Rich N. Two-tone suppression in the basilar membrane of the cochlea: mechanical basis of auditory-nerve rate suppression. J. Neurophysiol. 68:1087-1099, 1992.

Ruggero M, Rich N, Recio A, Narayan S, Robles L. Basilarmembrane responses to tones at the base of the chinchilla cochlea. J. Acoust. Soc. Am. 101:2151-2163, 1997.

Seluick PM, Patuzzi R, Johnstone BM. Measurement of basilar membrane motion in the guinea-pig using the Mossbauer technique. J. Acoust. Soc. Am. 72:131-141, 1982.

SHANNON RV. Psychophysical suppression of selective portions of pulsation threshold patterns. Hear. Res. 21:257-260, 1986.

Sommers MS, GeHr SE. Auditory suppression and frequency selectivity in older and younger adults. J. Acoust. Soc. Am. 103:1067-1074, 1998.

Stelmachowicz P, Lewis D, Larson L, Jesteadt W. Growth of masking as a measure of response growth in hearing-impaired listeners. J. Acoust. Soc. Am. 81:1881-1887, 1987.

van Klitzing R, Kohlrausch A. Effect of masker level on overshoot in running- and frozen-noise maskers. J. Acoust. Soc. Am. 95:2192-2201, 1994.

WEBER DL. Do off-frequency simultaneous maskers suppress the signal? J. Acoust. Soc. Am. 73:887-890, 1983.

Wegel RL, Lane CE. The auditory masking of one pure tone by another and its possible relation to the dynamics of the inner ear. Physics Rev. 23:266-285, 1924.

Wightman F, McGee T, Kramer M. Factors influencing frequency selectivity in normal and hearing-impaired listeners. In: Evans EF and Wilson JP (eds) Psychophysics and Physiology of Hearing. Academic Press, London, pp 341-350, 1977.

YASIN I, PLACK CJ. The effects of a high-frequency suppressor on tuning curves and derived basilar membrane response functions. J. Acoust. Soc. Am. 114:322-332, 2003.

Yates G, Winter I, Robertson D. Basilar membrane nonlinearity determines auditory nerve rate-intensity functions and cochlear dynamic range. Hear. Res. 45:203-219, 1990.

Zinn C, Maier H, Zenner H-P, Gummer AW. Evidence for active, nonlinear, negative feedback in the vibration response of the apical region of the in-vivo guinea-pig cochlea. Hear. Res. 142:159-183, 2000.

Zwicker E, Fastl H. Psychoacoustics-Facts and Models. SpringerVerlag, Berlin, 1990. 